\title{
La elaboración de modelos de competencias técnicas y su aplicación en la detección de necesidades formativas
}

\section{The development of technical competences models and their application for the detection of training needs}

\author{
Francisca Berrocal Berrocal \\ Universidad Complutense de Madrid (España) \\ ORCID: https://orcid.org/0000-0001-8426-0691 \\ fberroca@ucm.es
}

\section{NOTA BIOGRÁFICA}

Profesora Contratada doctora en la Facultad de Psicología de la Universidad Complutense de Madrid. Miembro del Grupo de Investigación Complutense "Evaluación y Desarrollo de las Personas y de las Organizaciones”. Sus principales líneas de investigación incluyen la gestión por competencias, la selección y evaluación de personas, así como la formación y desarrollo de personas.

Miguel Aurelio Alonso García

Universidad Complutense de Madrid (España)

ORCID: https://orcid.org/0000-0003-1584-4436

malonsog@ucm.es

\section{NOTA BIOGRÁFICA}

Profesor Contratado Doctor en la facultad de Psicología de la Universidad Complutense de Madrid (UCM). Dirige el Grupo de Investigación "Evaluación y Desarrollo de las Personas y de las Organizaciones" (GIDEPO) y el Programa de Mentoría de la UCM. Experiencia de treinta años en las áreas de Formación de Personal y Programas de Desarrollo de personas.

Raúl Ramírez-Vielma

Universidad de Concepción (Chile) ORCID: https://orcid.org/0000-0001-9231-4944 rauramir@udec.cl

\section{NOTA BIOGRÁFICA}

Profesor Asistente del Departamento de Psicología de la Universidad de Concepción. Sus principales líneas de investigación incluyen el diseño y re-diseño del trabajo en las organizaciones, la evaluación del desempeño, la reducción del estigma de salud mental en contextos laborales y el estudio de aspectos psicosociales del retiro laboral. Cuenta con experiencia profesional de doce años en consultoría de recursos humanos.

\section{RESUMEN}

La identificación de las competencias técnicas requeridas en las distintas áreas funcionales, por niveles profesionales, permite abordar los procesos de selección y formación de una forma más eficaz y eficiente, contribuyendo a la aportación de valor de las actuaciones desarrolladas por el Instituto Nacional de 
Administración Pública (INAP) a la consecución de los objetivos de la Administración General del Estado (AGE). El presente artículo tiene como objetivo proponer una metodología para identificar y definir las competencias técnicas necesarias para un correcto desempeño de los trabajos, así como contrastar su utilidad en la detección de necesidades formativas en los trabajadores de la AGE. La metodología utiliza grupos focales para la elaboración de perfiles en el área de gestión de personas y cuestionarios para la detección de necesidades de aprendizaje. Se describe el proceso de aplicación del análisis funcional llevado a cabo y los resultados obtenidos. En concreto, el modelo de competencias técnicas del área de gestión de personas para los roles de directivo, técnico y de apoyo, y los resultados del estudio de necesidades de formación realizado en el área. Se discuten las implicaciones del proceso y se formulan sugerencias de mejora en la detección de necesidades y la planificación de la formación dentro de la AGE.

\title{
PALABRAS CLAVE
}

Competencias técnicas; modelos de competencias; necesidades de formación; recursos humanos; análisis funcional.

\begin{abstract}
Identification of technical Competencies required in different functional areas, by professional levels, allows selection and training processes to be addressed in a more effective and efficient way, contributing to the added value of the actions developed by the National Institute of Public Administration (INAP) and achieving the General State Administration (AGE) objectives. The objective of this paper are to propose a methodology to identify and define the technical competencies needed for a correct work performance, as well as to contrast its usefulness in detecting training needs for AGE workers. The methodology uses both focus groups for the making of profiles in the area of people management and questionnaires to detect learning needs. Application process of the functional analysis carried out and the results obtained are described. Specifically, the model of technical competencies in the area of people management for managerial, technical and support roles, and the training needs study results carried out in the area. Implications of the process are discussed, and improvement suggestions for the detection of training needs and training planning within the AGE are formulated.
\end{abstract}

\section{KEYWORDS}

Technical competencies; competency models; training needs; human resources; functional analysis.

\section{SUMARIO}

1. INTRODUCCIÓN. 2. OBJETIVOS. 3. METODOLOGÍA. 3.1. PROCEDIMIENTO. 3.2. INSTRUMENTOS. 3.3. MUESTRA. 4. RESULTADOS. 4.1. MAPA DE COMPETENCIAS TÉCNICAS. 4.2. NECESIDADES DE FORMACIÓN. 5. CONCLUSIONES DISCUSIÓN Y SUGERENCIAS DE MEJORA. REFERENCIAS BIBLIOGRÁFICAS.

\section{INTRODUCCIÓN}

Una de las principales contribuciones que ha realizado la gestión de los recursos humanos contemporánea ha sido sensibilizar a las organizaciones acerca del importante rol que juegan las personas en cuanto al logro de sus objetivos estratégicos, ya no sólo en términos de eficacia y eficiencia, sino también en el necesario equilibrio que debe existir entre las necesidades de la propia organización y las necesidades de sus trabajadores.

El auge de los modelos por competencias puede explicarse en buena parte por el contexto en el que surgen. Actualmente, tanto las personas como las organizaciones, se encuentran ante un escenario caracterizado por un rápido ritmo de cambio en los requerimientos del trabajo, exigiendo un movimiento continuo, que posibilite superar los desafíos inherentes a unas condiciones de empleo adversas y volátiles (Feltovich, Spiro y Coulson, 1997; Hesketh y Neal, 1999). Los modelos por competencias han tenido una gran implantación debido a que facilitan o potencian comportamientos de alto desempeño y rendimiento asociados con la estrategia organizacional, utilizando un lenguaje contingente, operativo y propio de cada realidad organizativa (Campion et al., 2011; Macchi Silva et al., 2019; Salman et al., 2020), y que además cuentan con potencial para su aplicación en la gestión sustentable de las organizaciones (Galleli et al., 2019). 
En estos momentos, parece existir cierto consenso en considerar a las competencias como un conjunto de comportamientos observables directamente relacionados con un desempeño laboral óptimo (Blanco, 2007; Getha-Taylor et al., 2016; Pereda y Berrocal, 2011; Ramírez-Díaz, 2020). De hecho, diferentes autores en la materia coinciden en que al hablar de competencias se hace alusión a aquellas características personales que subyacen o determinan los comportamientos de un individuo y que están causalmente relacionadas con un rendimiento bueno o excelente en un trabajo o rol laboral (Boyatzis, 1982; Dalziel et al., 1996; Lévy-Leboyer, 1997; Pontnuru y Sahoo, 2016; Salman et al., 2020; Spencer y Spencer, 1993).

Hogg (1993) conceptualiza la competencia como las características de una persona que conducen a la demostración de habilidades y destrezas, que resultan en un desempeño efectivo dentro de un área ocupacional. La competencia también representa la capacidad de transferir habilidades y capacidades de un área a otra. Un análisis de la definición revela que:

- Las competencias son las características de una persona.

- Las competencias conducen a la demostración de habilidades y destrezas. Por tanto, la competencia debe ser observable.

- Las competencias deben conducir a un desempeño efectivo, que debe ser significativamente mejor que el de una persona sin ellas.

- La competencia también representa la capacidad de transferir habilidades y destrezas de un área a otra. Por ello, las competencias no pueden limitarse a un solo trabajo, sino que la persona debe ser capaz de llevarlas consigo.

En suma, se puede decir en términos semánticos que la competencia es, al mismo tiempo, un concepto relacionado con el trabajo (las tareas en las que una persona debe ser competente) y un concepto relacionado con la persona, que se expresa en un conjunto de comportamientos observables que conducen a un desempeño sobresaliente.

En una reciente revisión del estado del arte acerca de los modelos de competencias, Megahed (2018) identifica cinco grandes paradigmas de investigación: conductuales, comerciales, funcionales, educativos y situacionales.

En la noción de competencia técnica o profesional, objeto del presente estudio, adquieren especial protagonismo los enfoques funcionalista y educativo. El funcionalista describe un trabajo a partir de las funciones principales que llevan a los propósitos u objetivos que se deben conseguir, los elementos de competencia implicados y los criterios mínimos de ejecución, centrándose en los resultados reales del trabajo (Jackson, 2009), que permitan la certificación de la cualificación. El paradigma educativo define las competencias técnicas sobre la base del análisis funcional del rol, como las declaraciones de comportamiento y actitud (excluyendo los rasgos o creencias de personalidad) que se requieren para el desempeño del rol en el trabajo, y que generalmente se evalúan según el estándar comúnmente aceptado (Albino, 2018; Markus el al., 2005); a su vez, también se enfatizan tanto las especificidades de las tareas realizadas, la organización de las personas que las deben desempeñar (Gorriti y López, 2010), y las normas de competencia derivadas del análisis de las tareas laborales (Basoredo, 2008).

Las competencias técnicas son aquellas descripciones de lo que las personas tienen que saber (conocimientos) y saber hacer (habilidades) para desempeñar sus funciones de manera efectiva según los estándares y niveles de calidad establecidos por la organización. A su vez, las competencias técnicas pueden ser divididas en: a) específicas: son específicas de determinadas funciones laborales, trabajos o roles; b) transversales: son aquellas relevantes a distintas funciones laborales dentro de un área funcional, e incluso la organización.

Para la identificación y definición de las competencias técnicas adquiere un protagonismo especial el proceso de análisis funcional, una metodología desarrollada originalmente por Mansfield y Mitchell (1996) y Fine (1988), que se enfoca en los resultados del desempeño laboral para identificar el objetivo principal de la organización, área funcional o trabajo, dependiendo del nivel al que se esté trabajando, y a partir de él llegar a identificar la competencia. La característica principal de este modelo es que, además de centrarse en los resultados, identifica y describe de forma concreta las funciones claves y los fundamentos de la actividad laboral, lo cual permite la transferencia de las funciones hacia realidades laborales semejantes.

El enfoque funcional ha sido y es muy utilizado para facilitar el desarrollo de estándares ocupacionales nacionales, que se presentan en forma de un «mapa funcional», entendiendo como, «la representación gráfica del conjunto estructurado de las funciones laborales que se requieren para alcanzar los resultados previstos en una función global denominada propósito principal» (CONOCER, s. f., p. 3). 
En términos de la importancia de las competencias técnicas, se puede observar la necesidad de desplegar estrategias similares para la capacitación y formación permanente del personal en aquellas competencias que son específicas de unas áreas o ámbitos determinados. En este sentido, se intenta identificar y sistematizar aquellas competencias que suponen especialización (esto es, las competencias técnicas) que contienen un conjunto de conocimientos y habilidades que caracterizan la formación requerida y adquirida para desarrollar de forma excelente unas funciones determinadas en un área específica (Instituto Andaluz de Administración Pública, 2015).

En el ámbito de la formación los catálogos detallados de contenidos nivelados de las distintas competencias técnicas posibilitan el diseño de nuevas acciones formativas, basadas en una mejor identificación de las necesidades, en un mejor enfoque de la programación de las acciones formativas y con alto grado de consistencia entre los contenidos, métodos y medios didácticos. Asimismo, puede constituir el sustento del diseño de itinerarios formativos que supongan un salto cualitativo en la adaptación de la formación a las necesidades y en una mayor y mejor transferencia de los aprendizajes al desempeño en un puesto de trabajo (Instituto Andaluz de Administración Pública, 2015).

Para identificar las competencias técnicas existen al menos tres tipos de métodos que se pueden utilizar: el análisis funcional, el análisis ocupacional y el análisis constructivista. Dado el propósito del presente estudio, se desarrolla de forma más específica el análisis funcional, explicando de forma sintética las otras dos metodologías.

El análisis ocupacional es el proceso de recolección, ordenamiento y valoración de la información relativa a las ocupaciones, tanto en lo que se refiere a características del trabajo realizado, como a requerimientos que estas plantean al trabajador para un desempeño satisfactorio (Pujol, 1980). Uno de los métodos más destacados es el llamado método DACUM (DesignACurriculUM), que es una metodología de análisis cualitativo del trabajo que sigue la lógica del análisis ocupacional de tareas. Fue desarrollada con el fin de recolectar información sobre los requerimientos para el desempeño de trabajos específicos (Mertens, 1997). Por otra parte, el análisis constructivista, desarrollado fundamentalmente en Francia (Le Boterf, 2001; LévyLeboyer, 1997), pone el énfasis en la aportación de todos los trabajadores de la organización al proceso de construcción de la competencia, y no sólo del contenido de las funciones que se desarrollan, o de aquellos trabajadores más aptos. Las competencias se construyen como resultado de los procesos de aprendizaje, que se desarrollan entre todos los trabajadores, a partir del análisis de los problemas existentes en el contexto laboral (Mertens, 1997).

El análisis funcional toma como referente teórico los postulados de la escuela funcionalista de Sociología, y tiene sus orígenes en los esfuerzos de la administración del Reino Unido por adaptar los sistemas de formación y capacitación profesional a las necesidades del mercado laboral. Dentro de este enfoque, las organizaciones son entendidas como sistemas sociales abiertos en constante interacción con su entorno, pues su funcionamiento depende de sus relaciones con el mercado, la tecnología, las relaciones institucionales, y los fenómenos socioculturales, al mismo tiempo que la organización como sistema integra diferentes subsistemas interrelacionados funcionalmente (Mertens, 1997). Este enfoque define el concepto de competencia como un conjunto de conocimientos y cualidades profesionales necesarias para que el trabajador pueda desarrollar un conjunto de funciones y tareas que integran su trabajo u ocupación.

El análisis funcional es la herramienta principal utilizada para definir la naturaleza de un sector ocupacional y las funciones desempeñadas dentro de él. Este es un proceso esencial para definir la competencia laboral y establecer límites entre las diferentes ocupaciones. Un mapa funcional detallado nos permite establecer la contribución única de cada área ocupacional, lo que la hace diferente de todas las demás y, por tanto, su delimitación. Esto es esencial para garantizar que se identifiquen todas las funciones primarias (principales) y secundarias, que la relación entre ellas esté claramente establecida y se entienda la contribución directa que hacen al propósito global de la organización. Sin un mapa funcional no se puede decir dónde termina un área funcional y dónde comienza otra. El mapa funcional también permite, después de un proceso adecuadamente detallado de descifrar las funciones (conocido técnicamente como «desagregación»), llegar a un nivel de desglose que permite definir la competencia técnica de los trabajos y roles.

El análisis funcional no se refiere simplemente a los resultados en forma cuantificable, sino que se ocupa de los resultados más amplios que deben tener los ocupantes de los roles, abordando procesos como el desarrollo del personal, la retroalimentación y el seguimiento del desempeño, así como de las tareas.

Este enfoque permite acercarnos a las competencias técnicas de una función productiva, ya sea a nivel de sector de actividad, organización, área funcional o trabajo (CONOCER, 1998; Fletcher, 1997). 
El proceso de trabajo comienza estableciendo el objetivo o propósito principal de una función productiva, organización, área funcional o trabajo. En este estudio, área funcional y roles. Y con la ayuda de trabajadores expertos que conozcan bien el área en el que desempeñan su rol, mediante un proceso de trabajo sistemático, se construye el mapa funcional de ésta.

Para llevarse a cabo un análisis funcional deben respetarse tres principios (ChileValora y OIT, 2012):

1. De lo general a lo particular: una vez que se ha establecido el propósito clave (función principal) puede avanzarse en la especificación. El proceso se desarrolla hasta que se llega al nivel en el que los resultados necesarios para alcanzar la función precedente sean realizables por un trabajador. De esta forma se está dibujando un árbol de jerarquías que llega en su extremo más básico a las unidades y elementos de competencia. El resultado del análisis funcional se representa en un mapa funcional (ver Figura 1).

Figura 1. REPRESENTACIÓN ESTRUCTURAL DE UN MAPA FUNCIONAL

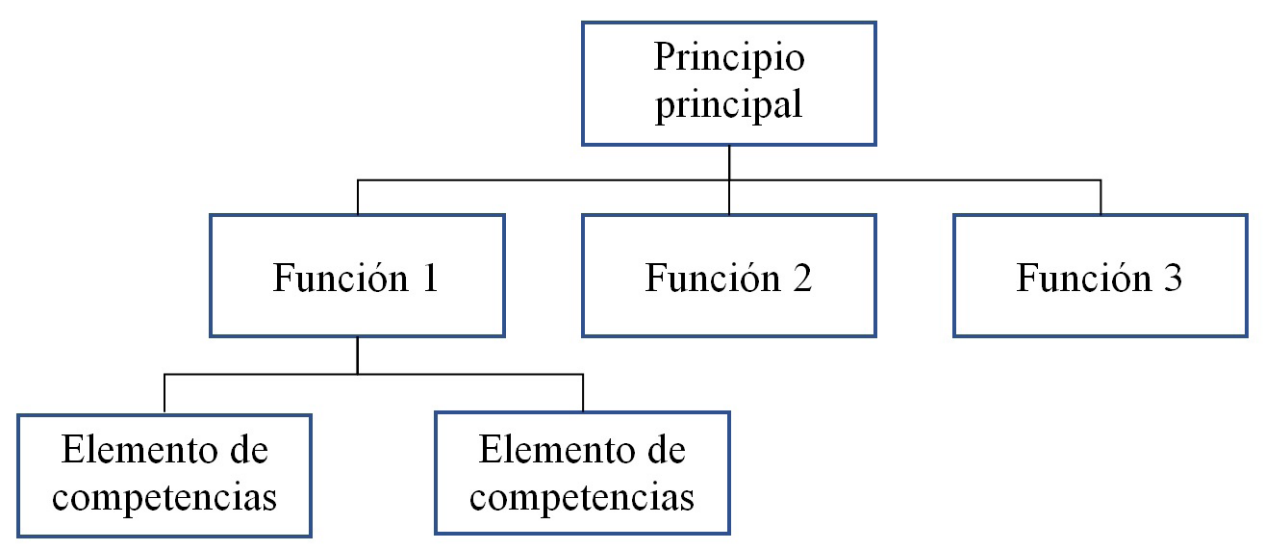

Fuente: Adaptado a partir de ChileValora y OIT (2012).

El número de niveles de desagregación dependerá del nivel de generalidad del que parta el análisis; es decir, viene condicionado por si su propósito es clarificar la función productiva de un sector de actividad, ocupación, empresa, área funcional o trabajo.

2. Funciones discretas: las funciones deben describirse de manera que permitan identificar de una manera comprensible y completa una acción de trabajo y un resultado que se debe alcanzar. Cada función debe poder ser identificada por su importancia, independientemente del contexto técnico. Por ejemplo, la función atender al usuario, puede describir el trabajo de un administrativo, de un recepcionista, entre otros.

3. La importancia de la redacción: la redacción debe seguir ciertas reglas que permitan mantener una estructura uniforme con el fin de conocer qué se hace, su lugar en la jerarquía y que se identifiquen con claridad los resultados.

Para los objetivos de este proyecto se utiliza el análisis funcional como metodología, ya que se consideró lo más adecuado, debido a que su empleo permite determinar las competencias técnicas a través de la identificación y ordenamiento de las funciones productivas, describiendo de manera precisa un área funcional desde su propósito principal hasta las contribuciones individuales requeridas para su cumplimiento (CONOCER, 1998).

Las unidades de competencia son similares a los grandes bloques de actividad del rol, mientras que los elementos de las competencias describen las actividades y comportamientos esperados de la persona que desempeña el trabajo, en otras palabras, lo que las personas deben poder hacer, contribuyendo así a la definición de estándares ocupacionales (Carroll y Boutall, 2011; Kopnov et al., 2018).

Como tal, cada unidad de competencia contiene un propósito del trabajo, o sea declaraciones completas de las competencias que incluye. Son la base para la certificación, a través de la evaluación, y el diseño curricular, a través de la formación.

Los elementos de una competencia forman las unidades de dicha competencia y determinan un resultado que el trabajador podrá obtener en el desempeño de una actividad laboral determinada.

Los resultados a obtener incluyen un criterio de calidad en el desempeño, que da cuenta del tipo de logro que se debe obtener y sus características, y permite evaluar la presencia de la propia competencia en un contexto físico (materiales, etc.) y organizativo (contexto, ambiente, etc.) determinado. 
Asimismo, con relación a dicho contexto, se especifican las situaciones y circunstancias en las que se desarrolla el elemento de la competencia y en las que debe obtenerse la evidencia de desempeño descrita, lo que también facilita su evaluación.

Las evidencias del desempeño especifican los resultados (productos, entregables, etc.), esenciales a verificar para contrastar cada elemento de la competencia y facilitar su evaluación. Los resultados pueden obtenerse por observación, ser evidencias o informaciones proporcionadas directamente por el trabajador, o referidas por aquellos a quienes afecta la competencia.

Cabe destacar que muchos estados han apostado por el análisis funcional como la herramienta principal para determinar la esencia del trabajo en los sectores ocupacionales, así como las funciones que se realizan dentro de él. El proceso permite determinar el conocimiento y las habilidades laborales y establecer límites entre las diferentes profesiones, trabajos o roles. De este modo, los mapas funcionales se convierten en un vínculo entre los estándares profesionales y los programas de formación específicos (Carroll y Boutall, 2011).

En este artículo se recoge parte de los resultados obtenidos en el proyecto "Elaboración de modelos de competencias técnicas en la Administración General del Estado" financiado por el INAP, referidos al área funcional de gestión de personas.

\section{OBJETIVOS}

El objetivo general del presente estudio es proponer una metodología que permita identificar y definir las competencias técnicas o profesionales necesarias para un correcto desempeño de los trabajos en los que es necesaria su aplicación, así como contrastar su utilidad en la detección de necesidades formativas de los ocupantes de los trabajos.

A partir de este objetivo general, se definieron los siguientes objetivos específicos:

- Elaborar el modelo de competencias técnicas del área de gestión de personas.

- Determinar las competencias técnicas para los roles de directivo, técnico y de apoyo del área.

- Contrastar la utilidad de los perfiles elaborados de los tres roles en la detección de las necesidades de aprendizaje de los ocupantes del área de gestión de personas.

\section{METODOLOGÍA}

La metodología para la elaboración de los perfiles de competencias técnicas se basa en la aplicación del análisis funcional y utiliza técnicas de carácter cualitativo, con la realización de grupos focales.

El análisis de necesidades de formación se basa en el cuestionario y el análisis de los resultados del mismo a través de técnicas cuantitativas.

\subsection{Procedimiento}

Para alcanzar los objetivos definidos, se siguieron las siguientes fases de trabajo:

\section{Recogida y análisis de información}

En primer lugar, se procedió a la recogida y análisis de información procedente de fuentes documentales internas a la AGE relacionadas con los objetivos, estructuras y actividades del área de gestión de personas, y distintos modelos de competencias procedentes de organizaciones privadas y públicas a nivel nacional e internacional.

En segundo lugar, se realizaron focus group con expertos, del área. El equipo de trabajo comenzaba la reunión explicando a los participantes que el objetivo general del estudio era recoger información que permitiría elaborar el modelo de competencias técnicas del área funcional y, de forma específica, elaborar el mapa funcional del área y determinar los conocimientos técnicos requeridos para el óptimo desempeño de las funciones incluidas en el rol. También se informaba que los resultados del estudio tendrían como aplicación práctica facilitar la detección de necesidades formativas, la elaboración del plan de formación adaptado a las necesidades de los trabajadores y, por consiguiente, mejorar la calidad de los procesos formativos. Asimismo, se informaba que los resultados podrían ser empleados en otros procesos de gestión de personas como la selección y el desarrollo de carreras. 
En cada uno de los grupos, se comenzaba pidiendo a los participantes que definieran el propósito principal del área; es decir, que explicaran para qué existe el área dentro de la AGE. Se recogían las aportaciones y se analizaban grupalmente hasta que se llegaba a un acuerdo consensuado del mismo.

A continuación, se explicaba el concepto de función dentro del análisis funcional y sus características y se solicitaba a los participantes que respondieran a la pregunta ¿qué hay que hacer para lograr el propósito principal del área desde el rol desempeñado?, y se indicaba que no se trataba de describir las tareas de un puesto de trabajo, sino de establecer las actividades que se desarrollan en el contexto del ámbito del rol en el que se llevan a cabo; esto es, desde el rol de directivo, técnico y/o apoyo.

Una vez establecidas las funciones principales se procedió a determinar los elementos de las competencias que deberían incluirse en cada una de las funciones principales. Se explicó qué eran los elementos de las competencias y los criterios que se debían utilizar para facilitar su estructuración (proceso secuencial, procesos o métodos, productos o resultados...). En esta fase, debido a la complejidad por la diversidad de funciones principales se formaron subgrupos en función de la experticia de los participantes en cada una de ellas. Para facilitar la tarea de los expertos, se les formulaba la pregunta, ¿qué comportamientos debe llevar a cabo un trabajador para realizar las actividades de su trabajo? Una vez recogidas las informaciones de los subgrupos, se procedió a poner en común la información con el objetivo de completar, matizar las informaciones, etc. Las respuestas permitirían elaborar los perfiles de competencias, formados por los comportamientos observables necesarios para desempeñar adecuadamente el trabajo. En la medida en que los comportamientos no los lleve a cabo adecuadamente se puede intervenir, a través de acciones de aprendizaje, por ejemplo.

Finalmente, se solicitaba a los participantes de focus group que indicaran los conocimientos específicos, los conocimientos sobre normativa y los referidos a las aplicaciones informáticas que se deben poseer para poder desarrollar de forma adecuada los elementos de las competencias de cada función principal.

A partir de este análisis de las respuestas proporcionadas por los distintos grupos focales, se elaboró el primer borrador de mapa funcional para cada uno de los niveles o roles incluidos en el estudio, que sería el punto de partida para las siguientes fases.

\section{Elaboración de los modelos de competencias a través de la información recogida}

En esta fase, en primer lugar, se envió vía correo electrónico a responsables del área de gestión de personas el borrador del mapa funcional y el listado de conocimientos (específicos, sobre normativa y de aplicaciones informáticas) elaborado por el equipo técnico en la fase previa, para su revisión y análisis individual, con el fin de realizar aportaciones de mejora. Posteriormente, se desarrolló otro focus group con responsables del área el cual estaba formado por expertos elegidos en función de su experticia en el área funcional, no en el desempeño de un rol determinado, ya que lo que se buscaba eran expertos con una visión global del área. Dichos expertos analizaron las propuestas y recomendaciones de los distintos miembros para llegar a un acuerdo consensuado del propósito principal, las funciones, elementos de las competencias y conocimientos.

\section{Detección de necesidades de aprendizaje}

El análisis de necesidades de formación se llevó a cabo a través de la aplicación de un cuestionario. Se envió un correo electrónico a los responsables de área de Recursos Humanos y se les pedía que lo cumplimentaran y lo compartieran con sus colaboradores para que también lo hicieran. El correo incluía un enlace a un formulario Google Forms que informaba sobre el estudio que la Universidad Complutense de Madrid (UCM) e INAP estaban realizando sobre las actividades en cada una de las áreas. Se informaba sobre el objetivo de la encuesta (facilitar la definición de las competencias técnicas de la Unidad y detectar posibles necesidades de aprendizaje), así como de la utilidad de los datos recogidos (elaborar el catálogo de competencias técnicas del área y facilitar que el INAP pueda elaborar su oferta de acciones formativas y desarrollo, teniendo en cuenta las necesidades detectadas por los propios ocupantes de los trabajos). Además, se informaba de la confidencialidad de los datos recogidos, así como del cumplimiento de la ley de protección de datos personales.

Los datos volcaban a una base de datos para su posterior análisis. 


\subsection{Instrumentos}

En cuanto a la estructura de cada grupo focal se abordaron los siguientes aspectos: presentación y objetivos del grupo, descripción del propósito principal del área de gestión de personas, extracción de las funciones principales a realizar para conseguir el propósito, determinación de las actividades y comportamientos para cada función principal, y definición de los conocimientos específicos necesarios para el desempeño eficaz del rol.

El cuestionario creado para analizar las necesidades de aprendizaje de cada rol dentro del área de gestión de personas recogía los siguientes aspectos:

- Datos descriptivos: en esta parte se realizaban preguntas relacionadas con el sexo, edad, nivel educativo, años de antigüedad en el puesto de trabajo, si tenía personas que dependieran jerárquicamente y el nivel dentro del AGE. También debían elegir, en función de las actividades que realizan en su trabajo, su rol, directivo, técnico o de apoyo.

- Áreas de actividad. Se presentaban las funciones principales (competencias técnicas) obtenidas del análisis funcional realizado en las primeras fases del proyecto, para que la persona señalara aquella/s en la/s que desempeña/n su trabajo en la actualidad.

- Actividades incluidas en cada función principal. Para cada una de las competencias técnicas, se listaban las actividades obtenidas en el análisis funcional para cada uno de los tres roles (directivo, técnico y de apoyo). Se solicitaba a cada participante que indicara, en una escala de 7 puntos, en qué medida consideraba que la formación podría serle útil para mejorar el dominio en dichas actividades ( 1 indicaba nada útil y 7 muy útil). En el caso de que no llevara a cabo la actividad en su trabajo, debía indicar la opción 0 «no es necesario para mi trabajo».

- Conocimientos. En este bloque los sujetos debían responder para cada uno de los conocimientos incluidos, en qué medida consideraba que necesitaría aprender más de la temática, en una escala tipo Likert de 5 grados ( 1 indicaba que no le sería nada útil aprender más y 5 que le resultaría muy útil). En el caso de que el ocupante del rol consideraba que el conocimiento no era necesario para su trabajo, debía seleccionar el grado «0». Los conocimientos se estructuraron en tres bloques: conocimientos específicos, conocimientos sobre normativa, y conocimientos sobre aplicaciones informáticas.

- Competencias genéricas. En esta última parte, se solicitaba a los sujetos que señalaran aquellas competencias que consideraban que les sería más útil desarrollar para lograr un mejor desempeño en su trabajo. El formulario recogía todas las competencias genéricas incluidas en el catálogo de competencias de la AGE, sin diferenciar en función del nivel del puesto que desempeñan las personas de la muestra o si éstas tienen personas a su cargo o no. Para las competencias señaladas, en una escala tipo Likert de 5 grados, debían indicar en qué medida consideraban que les resultaría útil desarrollar la competencia (1 indicaba que les sería poco útil desarrollar la competencia y 5 que les resultaría muy útil desarrollarla más).

\subsection{Muestra}

En un primer momento se realizaron tres grupos focales, cada uno de ellos formados entre 9 y 12 personas expertas en el área funcional de gestión de personas, un grupo para directivos, otro para técnicos y otro para personal de apoyo.

En una fase posterior se realizó otro grupo focal formado por 5 expertos que validarían la información elaborada a partir del análisis de las informaciones recogida a través de las fuentes documentales y los grupos focales de expertos en cada uno de los tres roles.

La muestra que cumplimentó el cuestionario estaba formada por 145, 20 de ellas directivos (13,8\%), 70 técnicos $(48,28 \%)$ y 55 de apoyo $(37,93 \%)$. El $41,38 \%$ eran hombres y el $58,62 \%$ mujeres. El promedio de edad de la muestra es de 50 años.

Las 145 personas pertenecían a 14 ministerios distintos de la AGE, lo que otorga heterogeneidad al estudio. El 78,6\% tienen estudios universitarios y la media de años de antigüedad en el trabajo actual en todos los roles es superior a los 5 años (rol de apoyo: 9,7 años; técnico: 8,1 ; y directivo: 5,1 ).

De las 145 personas que han cumplimentado la encuesta, el $50 \%$ indica que tiene personas a su cargo. El rol con mayor proporción corresponde al directivo (95\%), seguido del técnico (68,57\%). 
Con respecto al nivel dentro de la Administración General del Estado, los mayores niveles pertenecen al rol de directivo (entre el 28 y el 30 todos ellos), seguido por técnico y apoyo.

Las competencias del área de gestión de personas en las que desempeñan su trabajo las personas que han cumplimentado la encuesta, en función de su rol, se recogen en la Tabla 1. Como puede observarse, hay personas que realizan actividades en más de una competencia técnica.

TABLA 1. NúMERO DE PERSONAS POR ROLES QUE DESPEÑAN ACTIVIDADES DENTRO DE LAS DISTINTAS COMPETENCIAS TÉCNICAS DEL ÁREA DE GESTIÓN DE PERSONAS

\begin{tabular}{lcccc}
\hline \multicolumn{1}{c}{ Competencias técnicas } & Directivo & Técnico & Apoyo & Total \\
\hline Planificación de RRHH & 13 & 18 & 12 & 43 \\
\hline Selección y Provisión de Puestos & 11 & 22 & 18 & 51 \\
\hline Evaluación de Desempeño & 0 & 2 & 3 & 5 \\
\hline Formación y Desarrollo de Personas & 10 & 4 & 4 & 8 \\
\hline Gestión y Administración de Personal & 13 & 11 & 43 & 54 \\
\hline Relaciones Laborales & 9 & 3 & 3 & 6 \\
\hline Prevención de Riesgos Laborales & 0 & 1 & 2 & 3 \\
\hline
\end{tabular}

Fuente: Elaboración propia.

\section{RESULTADOS}

En este apartado, en primer lugar, se presenta el mapa de competencias técnicas del área de gestión de personas y los perfiles de competencias de los tres roles y, en un segundo lugar, las necesidades formativas detectadas.

\subsection{Mapa de competencias técnicas}

En función de las informaciones recogidas y analizadas a lo largo del estudio se definió el principio principal o misión del área de gestión de personas, así como 7 competencias técnicas (ver Tabla 2).

TABla 2. Principio general y Competencias técnicas del ÁRea de gestión de personas

\begin{tabular}{ll}
\hline \multicolumn{1}{c}{ Principio principal } & \multicolumn{1}{c}{ Competencias técnicas } \\
\hline Planificar, organizar y gestionar los RRHH con el fin de & • Planificación de RRHH. \\
asegurar que la organización cuenta con las personas & • Selección y Provisión de Puestos. \\
adecuadas para el desarrollo sus funciones de acuer- & • Evaluación de Desempeño. \\
do a la planificación estratégica de la organización, al & • Formación y Desarrollo de Personas. \\
marco jurídico aplicable a la gestión de Recursos Hu- & - Gestión y Administración de Personal. \\
manos (RRHH), los recursos económicos disponibles y & • Relaciones Laborales. \\
los cambios del entorno. & - Prevención de Riesgos Laborales.
\end{tabular}

Fuente: Elaboración propia.

A modo de ejemplo, se presentan los resultados de la competencia formación y desarrollo de personas, y se hace para los roles directivo (Tabla 3), técnico (Tabla 4) y apoyo (Tabla 5). El objetivo de la competencia formación y desarrollo es «impulsar y desarrollar la formación y el aprendizaje continuo de los empleados públicos con el fin de asegurar un desempeño eficaz, eficiente y seguro del trabajo, así como su motivación y satisfacción, de acuerdo con los objetivos y necesidades de la organización». 
TABLA 3. ACtIVIDADES Y CONOCIMIENTOS REQUERIDOS EN EL ROL DIRECTIVO

\begin{tabular}{|c|c|}
\hline Conocimientos & Actividades y comportamientos \\
\hline $\begin{array}{l}\text { - Conocimientos específicos: } \\
\text { - Elaboración de itinerarios } \\
\text { y modelos de desarrollo } \\
\text { profesional. } \\
\text { - Procesos e instrumentos } \\
\text { de detección } \\
\text { de necesidades } \\
\text { formativas: inmediatas y } \\
\text { estratégicas. } \\
\text { - La elaboración de planes } \\
\text { de formación anuales y } \\
\text { estratégicos. } \\
\text { - Formación y desarrollo } \\
\text { de personas: didáctica, } \\
\text { modalidades, } \\
\text { metodologías, } \\
\text { evaluación... } \\
\text { - Indicadores de calidad } \\
\text { de la formación. } \\
\text { - Marketing interno de } \\
\text { formación. }\end{array}$ & $\begin{array}{l}\text { a) Coordinar y supervisar el proceso de análisis de necesidades de formación, } \\
\text { determinando los métodos y fuentes de información más adecuados. } \\
\text { b) Anticipar posibles necesidades formativas (novedades normativas, de proce- } \\
\text { dimiento, tecnológicas, prioridades organizativas, cambios en el entorno, etc.) } \\
\text { que puedan afectar al desempeño del puesto de trabajo. } \\
\text { c) Priorizar las necesidades formativas inmediatas y estratégicas, en función de la } \\
\text { importancia y de la urgencia de las necesidades de las Unidades de la AGE, de } \\
\text { las personas y de acuerdo a criterios de eficacia y eficiencia. } \\
\text { d) Desarrollar programas de innovación en didáctica y provisión de actividades de } \\
\text { formación y desarrollo de personas. } \\
\text { e) Diseñar el plan de formación y supervisar su elaboración en función de las ne- } \\
\text { cesidades formativas detectadas y de acuerdo con criterios de calidad. } \\
\text { f) Definir las estrategias de difusión y comunicación del plan de formación. } \\
\text { g) Impulsar, coordinar y supervisar la implantación del plan de formación. } \\
\text { h) Establecer los criterios de selección de los participantes y del profesorado en } \\
\text { las acciones formativas. } \\
\text { i) Analizar y valorar los resultados del plan de formación y de las actividades for- } \\
\text { mativas desarrolladas con el fin de detectar necesidades no cubiertas y optimi- } \\
\text { zar futuras acciones. } \\
\text { j) Definir los criterios / indicadores de evaluación del impacto del plan de forma- } \\
\text { ción y las acciones formativas con el fin de conocer su eficacia, eficiencia y } \\
\text { calidad para identificar y proponer mejoras. } \\
\text { k) Comunicar los resultados y el impacto de los programas y proyectos de forma- } \\
\text { ción. } \\
\text { l) Asesorar estratégicamente a la alta dirección en materia de formación y desa- } \\
\text { rrollo de personas. } \\
\text { m) Establecer y mantener las relaciones necesarias con los distintos agentes impli- } \\
\text { cados en la consecución de los objetivos de la función. }\end{array}$ \\
\hline
\end{tabular}

Fuente: Elaboración propia.

TABLA 4. ACtIVIDADES Y CONOCIMIENTOS REQUERIDOS EN EL ROL DE TÉCNICO

\begin{tabular}{|c|c|}
\hline Conocimientos & Actividades y comportamientos \\
\hline $\begin{array}{l}\text { - Conocimientos específicos: } \\
\text { - La definición de exigencias de } \\
\text { formación de los puestos de } \\
\text { trabajo. } \\
\text { - El proceso y los instrumentos } \\
\text { de detección de necesidades } \\
\text { de formación y desarrollo: } \\
\text { inmediatas y estratégicas. } \\
\text { - La elaboración del plan de } \\
\text { formación anual y estratégico. } \\
\text { - Procedimiento de gestión de } \\
\text { gasto y presupuestaria. } \\
\text { - El diseño de acciones formativas } \\
\text { y las modalidades de formación. } \\
\text { - Indicadores de calidad de la } \\
\text { formación. } \\
\text { - Proceso e instrumentos de } \\
\text { evaluación de la satisfacción y } \\
\text { del impacto de los programas de } \\
\text { formación y desarrollo. }\end{array}$ & $\begin{array}{l}\text { a) Gestionar y coordinar el proceso de recogida y análisis de necesidades } \\
\text { formativas, proporcionadas por los distintos agentes implicados (traba- } \\
\text { jadores, unidades, etc.). } \\
\text { b) Participar en la realización del estudio económico de los programas de } \\
\text { formación para su posterior asignación presupuestaria. } \\
\text { c) Proponer la priorización de las necesidades formativas inmediatas y } \\
\text { estratégicas, en función de los objetivos marcados de formación y de- } \\
\text { sarrollo del personal de la organización y del estudio económico reali- } \\
\text { zado. } \\
\text { d) Elaborar las fichas de las acciones formativas. } \\
\text { e) Elaborar el plan anual de formación, gestionar su aprobación y comuni- } \\
\text { carlo a los agentes implicados. } \\
\text { f) Gestionar la disponibilidad de los medios necesarios para el desarrollo } \\
\text { de las actividades formativas. } \\
\text { g) Realizar la propuesta de selección de los participantes de las acciones } \\
\text { formativas en función del perfil de destinatarios establecido, así como } \\
\text { de selección de los docentes en función de los objetivos de la acción } \\
\text { formativa y de las características de los destinatarios. } \\
\text { h) Gestionar los bancos de recursos de aprendizaje de la unidad (material } \\
\text { didáctico, guías docentes, actividades, etc.). }\end{array}$ \\
\hline
\end{tabular}




\begin{tabular}{lll}
\hline \multicolumn{1}{c}{ Conocimientos } & \multicolumn{1}{c}{ Actividades y comportamientos } \\
\hline - Aplicaciones informáticas: & i) & $\begin{array}{l}\text { Participar en el diseño e implementación de los instrumentos de eva- } \\
\text { luación de la formación (satisfacción, aprendizaje, transferencia y resul- } \\
\text { tados) y en la gestión del proceso de evaluación. }\end{array}$ \\
$-\begin{array}{l}\text { Aplicaciones informáticas } \\
\text { propias: Módulo de Formación de }\end{array}$ & j)ealizar el seguimiento y evaluación continua del desarrollo de las ac- \\
SIGPE. & $\begin{array}{l}\text { ciones formativas y del plan de formación con el fin de detectar posibles } \\
\text { desviaciones y facilitar la toma de decisiones. }\end{array}$ \\
$-\begin{array}{l}\text { Aplicaciones informáticas de } \\
\text { propósito general (procesador } \\
\text { de textos, hoja de cálculo, }\end{array}$ & k) $\begin{array}{l}\text { Elaborar y proponer los manuales de procesos y procedimientos de la } \\
\text { función con fin de garantizar su adecuación a los criterios de calidad } \\
\text { PowerPoint...). }\end{array}$ & $\begin{array}{l}\text { establecidos y la normativa vigente. } \\
\text { Atender y resolver las consultas y dudas de los usuarios. }\end{array}$ \\
\hline
\end{tabular}

Fuente: Elaboración propia.

TABLA 5. ACtIVIDADES Y CONOCIMIENTOS REQUERIDOS EN EL ROL DE APOYO

\begin{tabular}{|c|c|}
\hline Conocimientos & Actividades y comportamientos \\
\hline $\begin{array}{l}\text { - Conocimientos específicos: } \\
\text { - Proceso de detección de } \\
\text { necesidades formativas. } \\
\text { - Proceso de elaboración de } \\
\text { programas formativos. } \\
\text { - Archivo. } \\
\text { - Aplicaciones informáticas: } \\
\text { - Aplicaciones informáticas propias } \\
\text { de gestión y registro del personal. } \\
\text { - Aplicaciones informáticas de } \\
\text { propósito general (procesador de } \\
\text { textos, hoja de cálculo, Acrobat...). } \\
\text { - Aplicaciones informáticas de control } \\
\text { económico y presupuestario. }\end{array}$ & $\begin{array}{l}\text { a) Apoyar administrativamente al proceso de detección de necesidades } \\
\text { formativas del personal, a través del envío y recepción de formula- } \\
\text { rios. } \\
\text { b) Apoyar en la selección de los participantes de las distintas acciones } \\
\text { formativas y en la selección de los formadores responsables de la } \\
\text { impartición de las acciones formativas. } \\
\text { c) Apoyar en la logística del desarrollo de las acciones formativas: pu- } \\
\text { blicación de los cursos que se van a impartir, selección y localización } \\
\text { de aulas, control de asistencia, entrega de material didáctico, recogi- } \\
\text { da de encuestas de satisfacción, emisión de certificados, gestión del } \\
\text { pago de las colaboraciones del profesorado, etc. } \\
\text { d) Analizar los resultados de las evaluaciones de satisfacción para faci- } \\
\text { litar la toma de decisiones. } \\
\text { e) Archivar la documentación relativa a las acciones formativas para } \\
\text { asegurar su disponibilidad. } \\
\text { f) Atender y resolver las consultas y dudas de los usuarios y, en caso } \\
\text { de necesidad, transferirlas al profesional más adecuado. }\end{array}$ \\
\hline
\end{tabular}

Fuente: Elaboración propia.

Además de los conocimientos incluidos en cada una de las competencias técnicas, para poder desempeñar de forma adecuada el rol dentro del área de gestión de personas, se requieren una serie de conocimientos sobre normativa que son transversales a los distintos roles: EBEP, convenio único, estatuto de los trabajadores, leyes de procedimiento administrativo aplicables, normativa y reglamentos específicos de la gestión de recursos humanos, ley de transparencia, acceso a la información pública y buen gobierno. y normativa de protección de datos.

\subsection{Necesidades de formación}

Las necesidades de aprendizaje detectadas a través del cuestionario elaborado están referidas a las actividades asociadas a cada competencia técnica, los conocimientos del área funcional y las competencias genéricas.

Para analizar las necesidades de formación relacionadas con las actividades a realizar en cada rol y competencia técnica, se ordenaron en función del grado de utilidad que han considerado los ocupantes de los roles que tendría para ellos recibir formación. Se han considerado necesidades formativas prioritarias, aquellas actividades que han recibido puntuaciones medias superiores a los 6 puntos sobre un máximo de 7 , necesidades secundarias las que han obtenido puntuaciones medias entre 4 y 6 puntos, y se ha considerado que no se requiere formación, en aquellas cuyas puntuaciones medias han sido inferiores a los 4 puntos.

Para el cálculo de la media y la desviación típica del grado de utilidad de la formación para mejorar el desempeño de las actividades, no se ha tenido en cuenta el «0», cuyo significado es que el participante consi- 
dera que la actividad «no es necesaria para su trabajo». El anclaje de «no es necesaria para su trabajo» (NN), permite conocer la pertinencia de incluir o no la actividad dentro de competencia para los distintos roles y; por tanto, la contrastación del modelo elaborado. En el caso de que el número de veces en el que una actividad sea considerada que no necesaria, sea elevado, nos estaría indicando que no es relevante en el rol en la actualidad.

A modo de ejemplo, se presentan los resultados obtenidos en la competencia Formación y Desarrollo de personas para el rol de directivos. Cuatro de las 13 actividades (Tabla 6) aparecen como prioritarias de cara a su inclusión en la planificación de acciones de formación y desarrollo, y las otras nueve como necesidades secundarias.

TABLA 6. UTILIDAD DE LA FORMACIÓN PARA INCREMENTAR EL DOMINIO DE LAS ACTIVIDADES de Formación y DesarRollo de Personas

\begin{tabular}{|c|c|c|c|c|}
\hline Actividades & M & DT & $\mathbf{n}$ & NN \\
\hline $\begin{array}{l}\text { Desarrollar programas de innovación en didáctica y provisión de actividades } \\
\text { de formación y desarrollo de personas. }\end{array}$ & 6,50 & 0,67 & 10 & 0 \\
\hline $\begin{array}{l}\text { Diseñar el plan de formación y supervisar su elaboración en función de las } \\
\text { necesidades formativas detectadas y de acuerdo con criterios de calidad. }\end{array}$ & 6,30 & 0,90 & 10 & 0 \\
\hline $\begin{array}{l}\text { Asesorar estratégicamente a la alta dirección en materia de formación y } \\
\text { desarrollo de personas. }\end{array}$ & 6,30 & 0,78 & 10 & 0 \\
\hline $\begin{array}{l}\text { Establecer y mantener las relaciones necesarias con los distintos agentes } \\
\text { implicados en la consecución de los objetivos de la función. }\end{array}$ & 6,30 & 0,64 & 10 & 0 \\
\hline $\begin{array}{l}\text { Definir los criterios / indicadores de evaluación del impacto del plan de } \\
\text { formación y las acciones formativas con el fin de conocer su eficacia, } \\
\text { eficiencia y calidad para identificar y proponer mejoras. }\end{array}$ & 5,90 & 1,45 & 10 & 0 \\
\hline Impulsar, coordinar y supervisar la implantación del plan de formación. & 5,70 & 1,42 & 10 & 0 \\
\hline $\begin{array}{l}\text { Anticipar posibles necesidades formativas (novedades normativas, de } \\
\text { procedimiento, tecnológicas, prioridades organizativas, cambios en el } \\
\text { entorno, etc.) que puedan afectar al desempeño del puesto de trabajo. }\end{array}$ & 5,60 & 1,20 & 10 & 0 \\
\hline $\begin{array}{l}\text { Priorizar las necesidades formativas inmediatas y estratégicas, en función } \\
\text { de la importancia y de la urgencia de las necesidades de las Unidades de la } \\
\text { AGE, de las personas y de acuerdo a criterios de eficacia y eficiencia. }\end{array}$ & 5,50 & 1,20 & 10 & 0 \\
\hline $\begin{array}{l}\text { Establecer los criterios de selección de los participantes y del profesorado } \\
\text { en las acciones formativas. }\end{array}$ & 5,50 & 1,69 & 10 & 0 \\
\hline $\begin{array}{l}\text { Coordinar y supervisar el proceso de análisis de necesidades de formación, } \\
\text { determinando los métodos y fuentes de información más adecuados. }\end{array}$ & 5,40 & 1,69 & 10 & 0 \\
\hline $\begin{array}{l}\text { Analizar y valorar los resultados del plan de formación y de las actividades } \\
\text { formativas desarrolladas con el fin de detectar necesidades no cubiertas y } \\
\text { optimizar futuras acciones. }\end{array}$ & 5,40 & 1,50 & 10 & 0 \\
\hline Definir las estrategias de difusión y comunicación del plan de formación. & 5,30 & 1,27 & 10 & 0 \\
\hline $\begin{array}{l}\text { Comunicar los resultados y el impacto de los programas y proyectos de } \\
\text { formación. }\end{array}$ & 5,30 & 1,49 & 10 & 0 \\
\hline
\end{tabular}

Fuente: Elaboración propia.

En la Figura 2 se comparan los roles de directivo, técnico y apoyo, para analizar de forma global las necesidades formativas en cada una de las siete competencias técnicas definidas. Todas las medias están por encima de 5 puntos sobre 7 , lo que indicaría que las personas de la muestra, consideran que recibir formación sobre las actividades de cada una de las competencias les resultaría útil. El rol de apoyo, de forma global, considera más útil recibir formación en todas las competencias, excepto en gestión administrativa de personal, que es precisamente el área donde más personas llevan a cabo sus actividades. 
Figura 2. MEdia de NeCESIDADES de FORMACIÓN POR COMPETENCIA TÉCNICA Y ROL

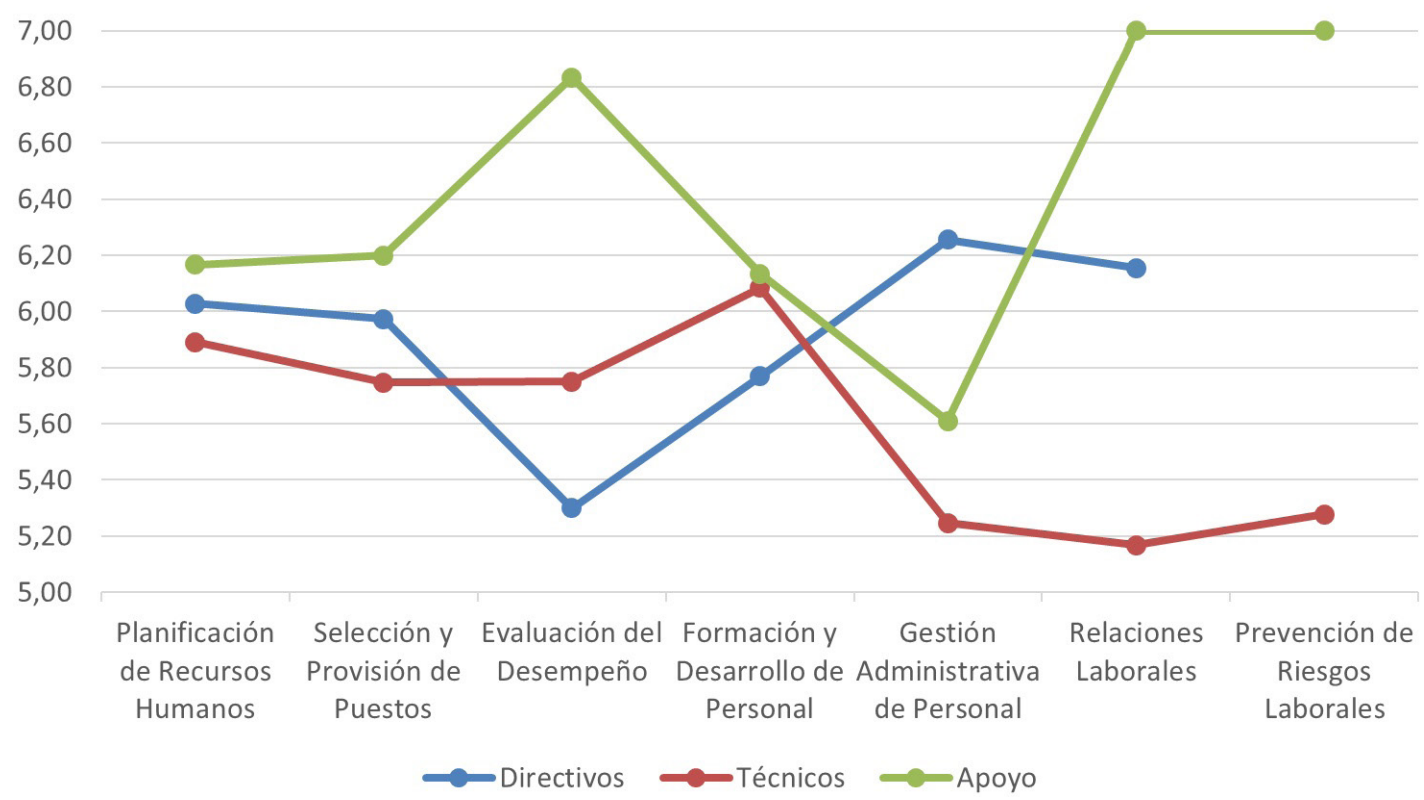

Fuente: Elaboración propia.

De la misma forma explicada para las actividades se han elaborado las tablas de resultados relativas a los conocimientos (específicos, sobre normativa y aplicaciones informáticas). Si bien, en este caso, debido a que la escala utilizada es de 5 niveles, se ha considerado un conocimiento como necesidad de formación prioritaria cuando la puntuación media ha sido mayor a los 4 puntos, necesidad secundaria entre 3 y 4 puntos, y que no se requiere formación, cuando la puntuación media ha sido inferior a los 3 puntos. Los directivos que han contestado la encuesta, consideran que hay 5 conocimientos específicos sobre los que deberían aprender más (Tabla 7) mientras que 12 serían necesidades secundarias. El conocimiento relacionado con la gestión de personas en las Administraciones Públicas ha sido señalado en todos los casos como no necesario. La última columna refleja la frecuencia de casos en los cuales no es necesario el conocimiento específico, frecuencia que en algunos casos alcanza el $50 \%$ de los casos.

En este punto, es importante resaltar que en la encuesta se presentaban de forma conjunta todos los conocimientos que se requieren para el desempeño adecuado de todas las competencias técnicas, debido a que hacerlo de otra forma implicaba la cumplimentación de un formulario muy largo y que además muchos de estos conocimientos serían repetitivos, ya que son comunes a varias competencias, sobre todos los referidos a normativas y aplicaciones informáticas. Teniendo en cuenta la diversidad de competencias del área y que los trabajadores pueden desempeñar sus actividades en una o más competencias, es lógico que se produzcan los resultados encontrados.

TABLA 7. Conocimientos ESPECíficos Y NECESIDADES DE APRENDIZAJE: DiRECTIVOS

\begin{tabular}{lcccc}
\hline \multicolumn{1}{c}{ Conocimientos específicos } & M & DT & $\mathbf{n}$ & NN \\
\hline Planificación estratégica y operativa en la Administración pública & 4,67 & 0,70 & 15 & 5 \\
\hline Diseño de procesos y técnicas de selección de personas & 4,43 & 1,05 & 14 & 6 \\
\hline Negociación colectiva & 4,23 & 0,80 & 13 & 7 \\
\hline $\begin{array}{l}\text { Evaluación de los resultados de las actuaciones de la gestión de recursos } \\
\text { humanos }\end{array}$ & 4,21 & 0,67 & 14 & 6 \\
\hline $\begin{array}{l}\text { Formación y desarrollo de personas: didáctica, modalidades, metodologías, } \\
\text { evaluación... }\end{array}$ & 4,07 & 0,80 & 14 & 6 \\
\hline
\end{tabular}


GAPP. Nueva Época - N. 26, julio 2021 - ISSN: 1989-8991 - DOI: https://doi.org/10.24965/gapp.i26.10813 - [Págs. 111-129]

La elaboración de modelos de competencias técnicas y su aplicación en la detección de necesidades formativas Francisca Berrocal Berrocal / Miguel Aurelio Alonso García / Raúl Ramírez-Vielma

\begin{tabular}{|c|c|c|c|c|}
\hline Conocimientos específicos & M & DT & $\mathbf{n}$ & NN \\
\hline Elaboración del cuadro de mando de recursos humanos & 4,00 & 1,13 & 14 & 6 \\
\hline Los sistemas de retribución fija y variable & 4,00 & 1,30 & 13 & 7 \\
\hline Elaboración de itinerarios y modelos de desarrollo profesional & 3,93 & 1,06 & 15 & 5 \\
\hline $\begin{array}{l}\text { Procesos e instrumentos de detección de necesidades de formación y } \\
\text { desarrollo }\end{array}$ & 3,86 & 1,36 & 14 & 6 \\
\hline La elaboración del plan de formación anual y estratégico & 3,85 & 1,17 & 13 & 7 \\
\hline Gestión de la RPT & 3,79 & 1,52 & 14 & 6 \\
\hline Indicadores de calidad de la formación & 3,77 & 0,97 & 13 & 7 \\
\hline Buenas prácticas en evaluación del desempeño & 3,57 & 0,90 & 14 & 6 \\
\hline Marketing interno de la formación & 3,57 & 1,05 & 14 & 6 \\
\hline Sistemas de reconocimiento del personal & 3,50 & 1,24 & 14 & 6 \\
\hline El plan de prevención de riesgos laborales & 3,10 & 0,83 & 10 & 10 \\
\hline Sistema de gestión de Prevención de Riesgos Laborales para la AGE & 3,00 & 0,77 & 10 & 10 \\
\hline La gestión de personas en la Administración pública & 0,00 & 0,00 & 0 & 0 \\
\hline
\end{tabular}

Fuente: Elaboración propia.

También se analizó, de forma global, la necesidad percibida por parte de los tres roles, de incrementar sus conocimientos (específicos, normativas y aplicaciones informáticas) (Figura 3). Todas las medias están por encima de 3 puntos sobre un máximo de 5 .

La mayor necesidad de formación en conocimientos específicos la tiene la muestra correspondiente al rol de directivos, con respecto a conocimientos sobre normativa y aplicaciones informáticas el rol de apoyo, seguido del rol de técnicos.

Figura 3. MEdia de NECESIDAdes de FORMACIÓN POR TIPO DE CONOCIMIENTOS Y ROL

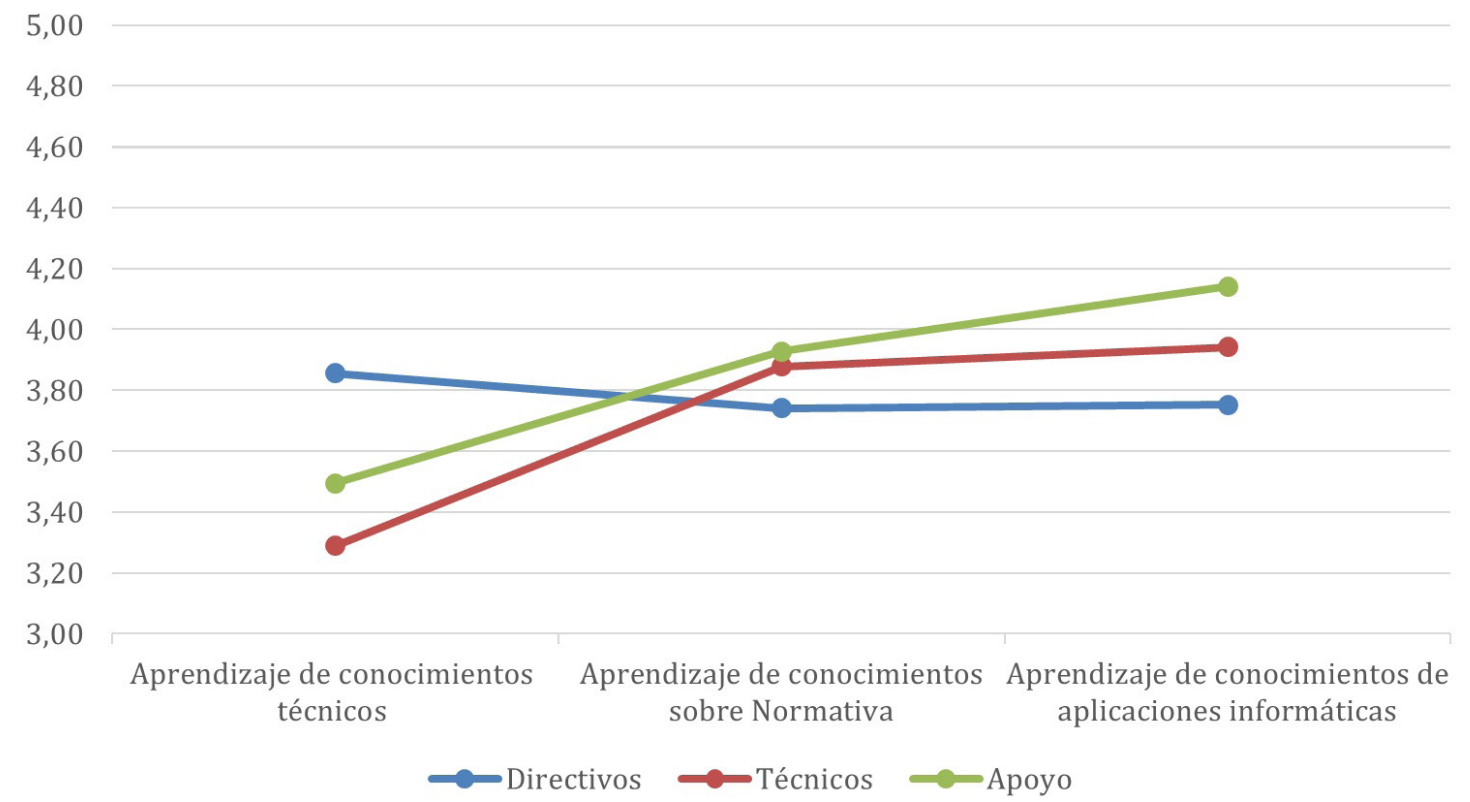

Fuente: Elaboración propia. 
Respecto a las competencias genéricas, en la Tabla 8 aparecen las puntuaciones medias de cada grupo. En gris oscuro se han señalado las competencias que cada uno de los grupos indican que tendrían necesidad de desarrollo prioritaria, en gris claro las secundarias y en blanco las que en la actualidad pueden considerarse no relevantes.

Los tres roles indican que las competencias aprendizaje continuo, comunicación, mejora continua, resistencia a la tensión, solución de problemas y trabajo en equipo y colaboración sería conveniente realizar acciones formativas para su desarrollo.

TABLA 8. MEdia de NeCESIDADES de FORMACIÓN POR COMPETENCIA GENÉRICA Y ROL

\begin{tabular}{lccc}
\hline \multicolumn{1}{c}{ Competencias genéricas } & Directivos & Técnicos & Apoyo \\
\hline Aprendizaje continuo. & 4,63 & 4,34 & 4,49 \\
\hline Compromiso con la AGE & 4,13 & 3,13 & 3,32 \\
\hline Comunicación & 4,79 & 4,26 & 4,21 \\
\hline Dirección de personas & 4,50 & 3,94 & 2,69 \\
\hline Flexibilidad y gestión del cambio & 4,63 & 3,92 & 3,86 \\
\hline Innovación & 4,38 & 4,02 & 3,57 \\
\hline Mejora continua & 4,46 & 4,18 & 4,18 \\
\hline Orientación a la calidad y a los resultados & 4,10 & 3,96 & 3,71 \\
\hline Orientación al ciudadano / usuario del servicio & 4,07 & 3,19 & 3,37 \\
\hline Orientación estratégica & 4,36 & 3,64 & 2,92 \\
\hline Planificación / organización & 4,71 & 4,16 & 3,98 \\
\hline Proactividad & 3,93 & 4,00 & 3,42 \\
\hline Resistencia a la tensión & 4,36 & 4,17 & 4,10 \\
\hline Solución de problemas & 4,43 & 4,31 & 4,02 \\
\hline Toma de decisiones & 4,31 & 4,19 & 2,98 \\
\hline Trabajo en equipo y colaboración & 4,50 & 4,34 & 4,21 \\
\hline
\end{tabular}

Fuente: Elaboración propia.

\section{CONCLUSIONES, DISCUSIÓN Y SUGERENCIAS DE MEJORA}

Del análisis de los resultados obtenidos con respecto a los modelos de competencias técnicas elaborados es posible extraer dos claras conclusiones:

a) El análisis funcional ha demostrado ser una metodología adecuada para la identificación de competencias técnicas, en un contexto como el de la AGE diverso y complejo, ya que ha permitido elaborar los mapas de competencias y determinar los modelos o perfiles de competencias y conocimientos técnicos de los tres roles incluidos en el estudio.

b) Las competencias y conocimientos técnicos incluidos en los modelos elaborados son necesarios para el adecuado desempeño de los trabajos. Todas las actividades incluidas en las 7 competencias técnicas se requieren en mayor o menor medida en los 3 roles. Este resultado nos estaría indicando la pertinencia de las competencias técnicas definidas y las actividades asociadas a las mismas.

La utilización de los modelos de competencias facilitaría la acreditación de competencias técnicas al permitir definir con claridad las exigencias de los trabajos en términos de las competencias y conocimientos técnicos necesarios para un adecuado desempeño laboral, lo que facilita el diseño de los procesos de 
selección, así como identificar las necesidades formativas, el diseño y el desarrollo de acciones formativas centradas en el aprendizaje y su transferencia al trabajo.

También consideramos que este es el inicio de un camino para ampliar la elaboración de mapas de competencias a otras áreas funcionales, así como modelos de competencias para perfiles o roles profesionales, e incluso trabajos clave para la AGE.

Los resultados sobre las necesidades de formación en las distintas competencias técnicas del área de gestión de personas ponen de manifiesto distintos aspectos útiles de cara a la planificación del plan formativo del INAP.

Los directivos perciben como útil la formación para incrementar su dominio en el desempeño de la mayor parte de las actividades planteadas en las distintas competencias técnicas, con puntuaciones iguales 0 superiores a 5 puntos (sobre un máximo de 7) en la mayor parte de los casos. Además, son muy pocos los casos en los que un comportamiento aparece señalado como «no relacionado con mi trabajo», lo que hace pensar que la necesidad afectaría a un elevado número de trabajadores.

Para fijar la necesidad como prioritaria se situó el punto de corte en 6 puntos, pero es un punto de corte arbitrario que puede moverse en función de los objetivos que el INAP se plantee en cuanto a número de acciones, personas a las que afecta, perfil profesional de las mismas, etc.

En lo relativo en la utilidad de recibir formación sobre conocimientos específicos, sobre normativa y aplicaciones informáticas, existe mayor diversidad. Es frecuente ver como un número significativo de personas señalan que no utilizan determinados conocimientos y como formarse en otros no les parece útil. No obstante, otros conocimientos sí son percibidos como relevantes para una parte importante de la muestra. Esto ocurre en el caso de los conocimientos sobre aspectos informáticos, donde los directivos por lo general no los perciben muy útiles para ellos.

Respecto a las competencias genéricas, la utilidad percibida por parte de los directivos, se sitúa en valores mayores de 4 puntos sobre 5 en la mayor parte de los casos. Parece que todas sean relevantes para ellos de cara a obtener una mayor cualificación.

Respecto al rol de técnicos, perciben como útil la formación para incrementar el dominio de las actividades en la mayor parte de las competencias, ejemplo de esto se da en planificación de recursos humanos, selección y provisión de puestos, evaluación de desempeño, y formación y desarrollo, con puntuaciones mayores a 5 puntos. En relaciones laborales y prevención de riesgos aparecen distintas actividades con puntuaciones de utilidad percibida entre 4 y 5 puntos, lo que indica que hay aspectos que parecen manejar lo suficientemente bien y no es necesario activar acciones formativas sobre los mismos.

En gestión administrativa de personal, la competencia en que más personas trabajan de la muestra, hay actividades que una parte importante de personas no las realizan en su trabajo, lo que lleva a pensar en que haya especialistas en determinados aspectos dentro del personal técnico que trabaja en dicha área.

El análisis de necesidades relativo a los conocimientos pone de manifiesto la baja utilidad que ven los técnicos en múltiples contenidos. Probablemente se debe a sus ya elevados conocimientos sobre aspectos necesarios para llevar a cabo su trabajo diario.

Respecto a las necesidades de formación o desarrollo relacionadas con competencias genéricas, son 9 competencias las que aparecen como prioritarias. Cabe resaltar que las competencias que parecen prioritarias para unas personas, otras afirman que no son necesarias para su trabajo. Esto pone de manifiesto la diversidad de competencias requeridas en función las actividades que realizan.

Por último, el personal de apoyo, es el que expresa una mayor necesidad de formación en las distintas competencias técnicas del área, con valores más altos en utilidad percibida de formación en un mayor número de actividades. La excepción se encuentra en el área de gestión administrativa de personal, en la que se dan los mayores valores de actividades que no se relacionan con su trabajo, quizá por la especificidad de los puestos dentro de esta competencia.

Es el colectivo que mayor utilidad ve al aprendizaje de conocimientos de aplicaciones informáticas.

Respecto a las competencias genéricas, discriminan claramente aquellas que desde su punto de vista tienen poca utilidad para ellos, como son las relacionadas con el rol directivo: dirección de personas, orientación estratégica, toma de decisiones... Es el colectivo con menos competencias genéricas que podrían marcarse como prioritarias, con tan solo seis.

Teniendo en cuenta la diversidad de competencias técnicas del área y que los trabajadores pueden desempeñar sus actividades en una o más competencias, es lógico que se produzcan los resultados encontrados.

El cuestionario y la forma de abordar los resultados del mismo, sienta las bases metodológicas para utilizarlo como instrumento de análisis de necesidades de formación. El INAP dispone de otros cuestionarios en 
línea para detectar necesidades de formación, basados en competencias genéricas, que podrían utilizarse de forma complementaria, ya que éste profundiza en los conocimientos y competencias técnicas específicas de un área funcional.

Pero si realmente se quiere que las informaciones recogidas a través del cuestionario sean útiles cara a la elaboración del plan de formación es conveniente poder individualizar las necesidades; esto es, poder identificar las necesidades de cada trabajador. Hacerlo facilitaría poder hacer convocatorias dirigidas a personas concretas (a las acciones formativas) y no en función de colectivos; así como realizar un seguimiento de las necesidades auto-percibidas, de la formación solicitada, de la asignada, y de la transferencia realizada al puesto de trabajo. Todo ello, contribuiría a mejorar la eficacia y la eficiencia de la formación, así como la satisfacción de los participantes.

Las necesidades de formación, a excepción de las estratégicas, son individuales; esto es, no presentan las mismas todos los ocupantes de un mismo puesto de trabajo. Definir necesidades de formación de forma colectiva, puede producir un descenso de la eficiencia y de la eficacia, puesto que se pueden estar dedicando recursos a formar a personas que no necesitan esa formación, mientras que es posible que no se les forme en lo que verdaderamente necesitan.

Incluir de forma directa a los trabajadores en la detección de sus necesidades formativas, permite conocer su visión sobre los principales obstáculos que tienen en su trabajo y que están dificultando lograr un óptimo desempeño en el mismo, así como de sus proyectos de desarrollo y cómo puede ayudarle la formación. Asimismo, es importante lograr la implicación de los individuos en su propia formación, ya que al comprobar que ésta responde a las necesidades que ellos mismos han detectado, mejorará los niveles de transferencia de los aprendizajes al puesto de trabajo y, por tanto, los resultados de la formación.

Estas informaciones deberían ser complementadas por las necesidades detectadas por los mandos y directivos en las personas que conforman sus equipos de trabajo. Los responsables podrán informar de las carencias que han detectado en el día a día en sus colaboradores, así como de los aspectos que son potencialmente mejorables en función de los objetivos y líneas de actuación definidos para sus Unidades y en qué medida la formación podría ayudar a conseguir los objetivos marcados. Para ello, sería conveniente diseñar instrumentos que permitan que los mandos y directivos informen de las necesidades formativas de sus Unidades o equipos de forma estructurada y sistemática.

El estudio no está exento de limitaciones, que es necesario precisar. La principal limitación ha sido el tamaño de la muestra. A pesar de que se hicieron dos recordatorios para que las personas cumplimentaran el cuestionario, y de que se envió a un gran número de ministerios y organismos, el número total de respuestas es limitado, lo que dificulta las posibilidades de generalizar los resultados.

Otro aspecto limitador son las dificultades para recoger los datos personales de los sujetos que cumplimenta el instrumento de detección de necesidades de formación. Hacerlo, permitiría conocer las necesidades formativas concretas de cada persona y, por tanto, poder individualizar la formación.

Por otro lado, en cuanto a implicaciones y sugerencias del estudio, es importante señalar que hacer participar a responsables y trabajadores debido al tamaño de la AGE y de los recursos del INAP, requiere un gran esfuerzo de recogida y procesamiento de las informaciones, así como de coordinación con los distintos Ministerios y Organismos, pero los beneficios que aportaría compensarían el esfuerzo realizado.

A estas fuentes directas se deberían sumar las informaciones procedentes de los resultados de gestión de los anteriores planes de formación, resultados de las selecciones de personal, de las evaluaciones del desempeño, etc.

Sin embargo, las fuentes especificadas permiten detectar necesidades a corto plazo y si realmente la función de formación quiere proporcionar un valor añadido a sus actuaciones, es necesario que trabaje de forma proactiva y se anticipe a posibles necesidades cara al futuro. Para ello, deberá abordar de forma sistemática la detección de necesidades estratégicas, e incluirlas dentro de su procedimiento de detección de necesidades de formación.

\section{REFERENCIAS BIBLIOGRÁFICAS}

Albino, G. (2018). Technical and behavioral competencies on performance evaluation: Petrek leaders' perspectives. SAGE Open, 8(2). https://doi.org/10.1177/2158244018780972

Basoredo Ledo, C. (2008). Diseño de currículos de formación a partir de tareas y por competencias [Tesis doctoral inédita]. Universidad de Deusto. 
GAPP. Nueva Época - N. 26, julio 2021 - ISSN: 1989-8991 - DOI: https://doi.org/10.24965/gapp.i26.10813 - [Págs. 111-129]

La elaboración de modelos de competencias técnicas y su aplicación en la detección de necesidades formativas

Francisca Berrocal Berrocal / Miguel Aurelio Alonso García / Raúl Ramírez-Vielma

Blanco Prieto, A. (2007). Trabajadores competentes: introducción y reflexiones sobre la gestión de recursos humanos por competencias. ESIC.

Bonder, A., Bouchard, C.-D. y Bellemare, G. (2011). Competency-based management. An integrated approach to human resource management in the canadian public sector. Public Personnel Management, 40(1), 1-10. https:// doi.org/10.1177/009102601104000101

Boyatzis, R. E. (1982). The competent manager: a model for effective performance. John Wiley \& Sons.

Campion, M. A., Fink, A. A., Ruggeberg, B. J., Carr, L., Phillips, G. M. y Odman, R. B. (2011). Doing competencies well: best practices in competency modeling. Personnel Psychology, 64(1), 225-262. https://doi.org/10.1111/j.17446570.2010.01207.x

Carroll, G. y Boutall, T. (2011). Guide to developing national occupational standards. UK Commission for Employment and Skills. https://assets.publishing.service.gov.uk/government/uploads/system/uploads/attachment_data/ file/304239/nos-guide-for-_developers-2011.pdf

ChileValora y OIT (2012). Guía de apoyo para la elaboración del análisis funcional. Centro Interamericano para el Desarrollo del Conocimiento en la Formación Profesional. Cinterfor/OIT. https://www.oitcinterfor.org/sites/default/ files/certificacion/ChileValora_GuiaApoyoAnalisisFuncional.pdf

Consejo de Normalización y Certificación de Competencia Laboral - CONOCER (1998). Análisis ocupacional y funcional del trabajo. Organización de Estados Iberoamericanos para la Educación, la Ciencia y la Cultura - OEI.

Consejo de Normalización y Certificación de Competencia Laboral - CONOCER (s. f.). Guía técnica para la elaboración del Mapa Funcional. Sistema normalizado de competencia laboral. Organización de Estados Americanos. http:// www.oas.org/udse/cd_trabajo/ingles/9-Gu\%EDa\%20mapa\%20funcional.pdf

Dalziel, M., Cubeiro, J. y Fernández, G. (1996). Las competencias: clave para una gestión integrada de los recursos humanos. Deusto.

Fine, S. A. (1988). Functional job analysis. En S. Gael (ed.), The job analysis handbook for business, industry and government. John Wiley \& Sons.

Feltovich, P. J., Spiro, R. J. y Coulson, R. L. (1997). Issues of expert flexibility in contexts characterised by complexity and change. En P. J. Feltovich, K. M. Ford y R. R. Hoffman (eds.), Expertise in context: human and machine (pp. 125-146). MIT Press.

Fletcher, S. (1997). Analysing competence: tools and techniques for analysing jobs, roles and functions. Kogan Page Publishers.

Galleli, B., Hourneaux Jr, F. y Munck, L. (2019). Sustainability and human competences: a systematic literature review. Benchmarking: An International Journal, 27(7), 1981-2004. https://doi.org/10.1108/BIJ-12-2018-0433

Getha-taylor, H., Blackmar, J. y Borry, E. L. (2016). Are competencies universal or situational? A state-level investigation of collaborative competencies. Review of Public Personnel Administration, 36(3), 306-320. https://doi. org/10.1177/0734371X15624132

Gorriti Bontigui, M. y López Basterra, J. (2010). Análisis de puestos de trabajo en la Administración Pública. Instituto Vasco de Administraciones Públicas - IVAP.

Hesketh, B. y Neal, A. (1999). Technology and performance. En D. R. Ilgen y E. D. Pulakos (eds.), The changing nature of performance: implications for staffing, motivation and development (pp. 21-55). Jossey-Bass.

Hogg, B. (1993). European Managerial Competencies. European Business Review, 93(2), 21-26.

Instituto Andaluz de Administración Pública (2015). Diccionario de competencias técnicas de la Administración General de la Junta de Andalucía. Junta de Andalucía.

Jackson, D. (2009). Profiling industry-relevant management graduate competencies: the need for a fresh approach. International Journal of Management Education, 8(1), 85-98.

Kopnov, V. A., Shmurygina, O. V., Shchipanova, D. E., Dremina M. A., Papaloizou L., Orphanidou, Y. y Morevs, P. (2018). Functional analysis and functional maps of qualifications in ECVET context. The Education and Science Journal, 20(6), 90-117. https://doi.org/10.17853/1994-5639-2018-6-90-117

Le Boterf, G. (2001). Ingeniería de las competencias. Gestión 2000.

Lévy-Leboyer, C. (1997). Gestión de las competencias: cómo analizarlas, cómo evaluarlas, cómo desarrollarlas. Gestión 2000.

Macchi Silva, V. V., Ribeiro, J. L. D., Álvarez, G. R. y Caregnato, S. E. (2019). Competence-based management research in the web of science and scopus databases: scientific production, collaboration and impact. Publications, 7(4), 60. https://doi.org/10.3390/publications7040060

Mansfield, R. y Mitchell, L. (1996). Towards a competent workforce. Gower.

Markus, L. H., Cooper-Thomas, H. D. y Allpress, K. N. (2005). Confounded by competencies? An evaluation of the evolution and use of competency models. New Zealand Journal of Psychology, 34(2), 117-126.

Megahed, N. (2018). A Critical review of the literature and practice of competency modelling. Sustainability and Resilience Conference: Mitigating Risks and Emergency Planning. KnE Social Sciences, 3(10), 104-126. https:// doi.org/10.18502/kss.v3i10.3106

Mertens, L. (1997). Competencia laboral: sistemas, surgimientos y modelos. Centro Interamericano para el Desarrollo del Conocimiento en la Formación Profesional - CINTERFOR/OIT. 
Pereda, S. y Berrocal, F. (2011). Dirección y gestión de recursos humanos por competencias. Editorial Universitaria Ramón Areces.

Potnuru, R. K. G. y Sahoo, C. K. (2016). HRD interventions, employee competencies and organizational effectiveness: an empirical study. European Journal of Training and Development, 40(5), 345-365. https://doi.org/10.1108/ejtd-022016-0008

Pujol, J. (1980). Análisis ocupacional. Manual de aplicación para instituciones de formación. Centro Interamericano para el Desarrollo del Conocimiento en la Formación Profesional - Cinterfor/OIT.

Ramírez-Díaz, J. (2020). An approach by competencies and its current relevance: considerations from occupational guidance in educational context. Revista Electrónica Educare, 24(2). https://doi.org/10.15359/ree.24-2.23

Salman, M., Ganie, S. A. y Saleem, I. (2020). The concept of competence: a thematic review and discussion. European Journal of Training and Development, 44(6/7), 717-742. https://doi.org/10.1108/EJTD-10-2019-0171

Spencer, L. M. y Spencer, S. M. (1993). Competence at work models for superior performance. John Wiley \& Sons. 